\title{
Fintech de crowdfunding: retos de su desarrollo en Perú
}

Yamile Cárdenas del Río

Abogada por la Pontificia Universidad Católica del Perú.

Katia Vásquez Reyes

Abogada por la Pontificia Universidad Católica del Perú.

SUMARIO:

I. Introducción.

II. Principales ventajas y retos de las fintech.

1. Efectos en el sector financiero.

III. Impacto en las tasas de interés.

IV. Regulación de fintech en otras legislaciones.

1. Caso España.

2. Caso México.

3. Caso Chile y Colombia.

V. El desafío de la SMV.

VI. Conclusiones. 


\section{RESUMEN:}

El desarrollo de las fintech a nivel mundial es inminente. Sin embargo, el Perú aún no cuenta con una regulación que promueva su ingreso y dinamice el sector. El dictamen que regula el financiamiento participativo todavía se encuentra pendiente de aprobación en el Congreso y, de aprobarse, los mayores retos los tendrá la Superintendencia del Mercado de Valores (SMV). Una mayor inclusión financiera con impactos positivos en la economía todavía parece lejana, pero se está iniciando.

Palabras clave: fintech, crowdfunding, tasas de interés, Superintendencia Nacional del Mercado de Valores, SMV, sector financiero, financiamiento participativo, regulación.

\footnotetext{
ABSTRACT:

Worldwide fintech development is imminent. Nevertheless, Peru still does not have regulation that encourages the entry of fintechs to the Peruvian market and invigorates the sector. The bill that regulates crowdfunding is yet pending approval form Congress. If approved, the biggest challenges will be faced by the Peruvian Superintendence of Securities Market (Superintendencia del Mercado de Valores-SMV). A bigger financial inclusion with positive impact in the economy still seems distant, but it is starting.

Keywords: contractual obligations, compulsory relationships, undue payment, civil liability, excess payment, unjust enrichment.
}

\section{INTRODUCCIÓN}

La propuesta de regulación de fintech en el Perú está en marcha, pero todavía es limitada. Su generalidad es positiva, al asemejarse a otras regulaciones, pero de los detalles que se definirán en el reglamento, dependerá el desarrollo del sector en el país.

La industria fintech ha tenido un rápido crecimiento y en el mundo se presenta como un fenómeno sin freno.

"Hace unos pocos años, la innovación tecnológica tocó la puerta del mundo financiero. La tecnología ahora no solo se centra en la transmisión de información, sino que también ha encontrado formas de mejorar la prestación de servicios financieros, haciéndola más rápida, segura e inclusiva. Las startups, dedicadas a la tecnología financiera, son conocidas ahora como Fintech -Financial Technology -"

En mayo del 2019 el Poder Ejecutivo presentó al Congreso un proyecto de ley con la finalidad de regular y supervisar el financiamiento par- ticipativo y a las sociedades que administran estas plataformas enfocadas en crowdfunding financiero, es decir, plataformas que conectan a receptores con inversionistas con la finalidad de financiar un determinado proyecto.

La iniciativa, hasta el momento de la redacción de este artículo - septiembre 2019-, cuenta con dictamen aprobatorio de la Comisión de Economía del Congreso. Éste regula la actividad y fija obligaciones y prohibiciones a las empresas que van a gestionar las plataformas. No obstante, se deja algunos elementos para una regulación posterior, teniendo en cuenta la mayor flexibilidad posible. En la medida en que esta industria vaya creciendo, en el camino el regulador podrá hacer los ajustes.

Es claro que mediante la tecnología también se puede tener acceso a créditos con las mismas garantías que a través de una institución financiera y, sin embargo, las sociedades que se encargan de ello no cuentan con una regulación que las dote de mayor seguridad. Además, los costos tradicionales asociados al financiamiento se ven reducidos con el uso de estas nuevas herramientas.

1. Adolfo Morán y Sergio García, «Criptomonedas y psicología financiera», Revista Themis (2019): 246. 
Una adecuada regulación del crowdfunding, en definitiva, alentaría la inversión. Si funciona, los distintos tipos de crowdfunding podrían desarrollarse. Así tenemos por ejemplo, el crowdfunding de recompensa, que implica compartir una idea con personas que estén interesadas en desarrollar un proyecto, para luego devolver las recompensas en forma de producto. El crowdfunding de inversión, sin embargo, apunta a inversiones más grandes. Éste fue el caso de Glovo, en España, quienes para iniciar requerían de un capital inicial de aproximadamente 300,000 euros. Las personas que aportaron multiplicaron diez veces su inversión.

Por otro lado, el crowdfunding inmobiliario tiene como finalidad financiar proyectos del sector inmobiliario, por lo que genera grandes ingresos de dinero. Finalmente, el crowdfunding de préstamo es utilizado por las empresas que buscan diversificar sus fuentes de financiamiento, a través de entidades que ofrecen una tasa inferior a la que ofrecen los bancos.

Si bien el dictamen aprobado el mes pasado por la Comisión de Economía del Congreso busca volver al segmento más atractivo al darle mayor predictibilidad y garantías a los inversionistas, "se requiere un reglamento más específico y modificaciones normativas para que se logre el objetivo"2.

\section{PRINCIPALES VENTAJAS Y RETOS DE LAS FINTECHS}

Cada vez más vemos un constante crecimiento de las startups ${ }^{3}$ que aprovechan las desarrollos tecnológicos para brindar una oferta de servicios financieros acordes con el mercado innovador. Estas empresas cuentan con varios in- centivos para insertarse en este nuevo mercado en desarrollo.

No solo nos referimos al ahorro económico que se genera al trabajar mediante una plataforma virtual, sin tener el gasto habitual de arrendamiento de oficinas, mantenimiento, pago de personal de vigilancia, limpieza u otros gastos administrativos no ligados directamente al giro del negocio. Sino también que se genera un ahorro de tiempo, puesto que aquello que normalmente bajo un procedimiento burocrático y sujeto a una serie de aprobaciones internas de un banco puede demorar horas o incluso días, mediante una plataforma de financiamiento tecnológico altamente especializada, se puede generar en cuestión de minutos o incluso segundos. $Y$ este ahorro de tiempo se traduce a su vez en un ahorro adicional de recursos y de horas-hombre $-\mathrm{HH}-$.

Otro factor importante que juega a favor de las fintech es que, al funcionar bajo un esquema tecnológico especializado, se puede gestionar de manera más transparente la empresa, en comparación con lo que sucede en las entidades bancarias donde es imposible controlar todos los comportamientos que no necesariamente se efectúan a nivel informático. Mientras mayor es el grado de sistematización, mayor es el grado de transparencia y de eficiencia ${ }^{4}$.

Una razón importante que también fomenta el surgimiento de fintech es la posibilidad de obtener un alto rendimiento al brindar servicios financieros digitales innovadores, sin tener que cumplir todos los requisitos y seguir los procedimientos que nuestra legislación impone a las empresas del sistema financiero, puesto que se tratan de empresas que manejan capi-

2. Yamile Cárdenas, «Crowdfunding: regulación limitada», SEMANAeconómica, n. 1679 (2019).

3. Para efectos del presente artículo, entendemos por "startups" a aquellas empresas creadas recientemente que comercializan productos y/o servicios mediante el uso intensivo de las tecnologías de la información, sobre la base de un modelo de negocio de crecimiento rápido y sostenido en el tiempo — para mayor información ver definición indicada en https://economiatic.com/que-es-una-startup/—.

4. Rosa Castelnou, 28 de octubre de 2019, «Los beneficios de la tecnología "fintech" para tu empresa», Blog Captio by emburse, https://www.captio.net/blog/beneficios-de-la-tecnologia-fintech-para-tu-empresa\#ventajas. 
tales muy inferiores y que están aún en etapa de maduración. Como veremos más adelante la propuesta de regulación de las fintech establece parámetros generales, pero por el momento no profundiza en su reglamentación específica, lo cual nos parece adecuado para el rol que tienen actualmente en el Perú.

Del otro lado, tenemos a los inversionistas que apuestan por las fintech, al considerarlas más flexibles que otras entidades financieras, más transparentes y rápidas de usar, en comparación con las colas que se deben hacer en un banco para solicitar un préstamo o efectuar una inversión, o la sujeción a los horarios preestablecidos e inamovibles que tienes las empresas financieras.

Es así que "la financiación fintech ha contribuido a mejorar el acceso al mercado de personas y empresas subatendidas desde el punto de vista financiero, además de brindar opciones adicionales a los inversores"s.

Debemos resaltar que el público objetivo de las fintech se encuentra conformado en parte por clientes "subancarizados", es decir, por clientes que pueden tener cuentas de ahorro o tarjetas de débito, pero no han asumido deudas bancarias $^{6}$. Es precisamente a estas personas quienes muchas veces perciben a las fintech como una idea innovadora, más atractiva y adaptable a sus horarios y requerimientos específicos.

Es cierto también que las fintech son preferidas por un grueso de la población menos conservador y más moderno, por lo que no terminan estando dirigidas realmente a todo el público en general. No obstante, eso no genera mayores inconvenientes para el desarrollo de este tipo de mercados, porque como vemos todo el tiempo estamos evolucionando como sociedad y adaptándonos a un mundo globalizado, en el que cada vez más las generaciones estarán inmersas en un desarrollo tecnológico. Por esto, especialmente a futuro, hay grandes expectativas de crecimiento de las fintech.

Dentro de los principales servicios que brindan las fintech en el mundo, está el financiamiento participativo o crowdfunding, que ha tenido bastante éxito como mecanismo de obtención de financiamiento para la ejecución de proyectos, bajo las diversas modalidades que existen, tales como recompensa, préstamo, inversión y otras. Justamente por ello, se propone en Perú regular esta modalidad de financiamiento.

Si bien es evidente que las fintech tienen un futuro prometedor y acarrean varias ventajas como las indicadas, no se debe dejar de lado la existencia de determinados riesgos financieros inherentes a las operaciones. Al igual que con las demás entidades financieras, las fintech requieren también contar con un respaldo, de modo que se proteja a los usuarios ante cualquier pérdida por un mal manejo de los fondos por parte de las fintech. Por ello, se propone que para ser autorizada por la Superintendencia del Mercado de Valores-SMV, toda plataforma de financiamiento participativo debe contar con una póliza de seguro de cubra el riesgo de deshonestidad, siendo que el monto de la póliza dependerá del monto del proyecto. A mayor monto del proyecto, mayor deberá ser la cobertura del seguro, ya que mayor será el riesgo de inversión.

Bajo la misma línea es que se permite en la legislación española que en caso las plataformas de financiación participativa no dispongan de un capital mínimo de 60,000 euros, deberán

5. Stijn Claessens, Jon Frost, Grant Turner y Feng Zhu, «Mercados de financiación fintech en todo el mundo: tamaño, determinantes y cuestiones de política», Bank for International Settlements (2018): 18, https://www.bis.org/publ/ qtrpdf/r_qt1809e_es.pdf.

6. Lorena Guarino, 11 de febrero de 2019, «Guerra de Beneficios: cómo los bancos y las fintech compiten para ganar más clientes en la Argentina», iProUp, https://www.iproup.com/mundo-fintech/2610-descuento-promocion-banco-digital-Bancos-vs-fintech-que-beneficios-otorgan-para-atraer-clientes. 
tener un seguro de responsabilidad civil profesional, un aval u otra garantía equivalente que permita hacer frente a la responsabilidad por negligencia en el ejercicio de su actividad profesional; o tener una combinación de capital inicial y de seguro de responsabilidad civil profesional, aval u otra garantía similar?. Todo esto apunta a la protección del consumidor.

Otro riesgo también inherente a las operaciones de las fintech es el riesgo cibernético como por ejemplo la posible existencia de ciberataques o fraudes en red. Si bien es cierto que existe un incremento en este tipo de ataques, también es verdad que frente a estos escenarios surgen constantemente soluciones de seguridad enfocadas en la prevención, y específicamente en la identidad digital, ya que resulta fundamental que las plataformas de financiamiento -aplicaciones, páginas web, etc.- cuenten con un historial crediticio de los actores involucrados en este tipo de operaciones ${ }^{8}$.

Incluso ante dicho escenario de inseguridad, actualmente se observa en América Latina que "dos de cada tres empresas en los segmentos de pagos, banca digital, y puntaje crediticio, seguridad y fraude cuentan ya con planes de contingencia en casos de incidentes y/o seguros de ciberseguridad"9. Por lo que podemos afirmar que existe preocupación por minimizar dicho riesgo.

\section{Efectos en el sector financiero.}

La dinamización del mercado financiero dependería de una adecuada regulación de fintech de crowdfunding. Como ya se mencionó anteriormente, todavía la propuesta para regular estas plataformas es limitada y general. La expectativa que se tiene sobre las medidas que el ente regulador pueda aplicar son grandes.
La próxima regulación de empresas de crowdfunding favorecería el ingreso de más empresas de este segmento. Sin embargo, para que los beneficios esperados se concreten-menores tasas de interés y mayor competencia- no solo se requieren mayores precisiones del regulador, sino que también se hacen necesarias algunas modificaciones normativas.

De acuerdo con Emprende UP - Centro de emprendimiento e innovación de la Universidad del Pacífico-, se han identificado 130 fintech en el país, de las cuales solo el $4 \%$ realizaría actividades de crowdfunding, pues, según el texto de la ley, el financiamiento debe llevarse a cabo necesariamente a través de la plataforma y la mayoría no cumple con este requisito.

Según el dictamen, las empresas que realizan crowdfunding en el Perú son Afluenta, Independencia, Prestamype y Salven. Sin embargo, para la elaboración de un artículo publicado en SEMANAeconómica, tuvimos la oportunidad de conversar con los CEOs de Prestamype e Independencia, quienes indicaron que no estarían sujetas a la próxima regulación. En el caso de Prestamype, el hecho de no conectar a una persona con varios inversionistas, sino solo con uno, implicaría su no consideración como empresa de crowdfunding. Por su parte, Independencia, si bien otorga préstamos, lo hace con fondos propios, lo cual la excluiría de la regulación.

En cuanto a los efectos específicos en el sector financiero, no puede dejarse de lado el hecho de que existe necesariamente una estrecha relación entre las ya establecidas empresas del sistema financiero y las fintech de crowdfunding, siendo que dicha relación debe ser de cooperación, en tanto que las fintech permiten complementar la

7. Para mayor información ver el artículo 56 de Ley 5/2015, del 27 de abril, de fomento de la financiación empresarial.

8. «Fintech. América Latina 2018. Crecimiento y consolidación», Banco Interamericano de Desarrollo, https://www. nodoka.co/apc-aa-files/319472351219cf3b9d1edf5344d3c7c8/fintech-amrica-latina-2018-crecimiento-y-consolidacin-1-.pdf.

9. Ibíd. 
oferta de servicios que tienen los bancos. Al respecto, Gemma Ames nos dice lo siguiente:

"A pesar de que las fintech argumentan ofrecer servicios superiores a los que ofrecen los bancos, sustentándose en su agilidad de innovación y comprensión de las tecnologías actuales que le permite entender mejor $y$ ajustarse a las necesidades de los usuarios. No obstante, en el contexto de la teoría de intermediación financiera no son competitivos debido a que, en el caso de los préstamos en línea, solo podrían reducir mejor las fricciones de financiamiento en el mercado crediticio, pero no pueden competir con los bancos en la provisión de servicios de liquidez y en la transformación de activos ${ }^{\prime \prime 0}$.

No obstante, el entendimiento de que estamos ante servicios que pueden convivir en el mercado no siempre fue así. De hecho, cuando las fintech comenzaron a surgir en el mercado financiero, hace un par de años, no en todos los casos tuvieron la mejor respuesta por parte de los bancos. Por ejemplo, en la experiencia chilena, hubo una primera etapa de rechazo absoluto de las fintech"1.

Afortunadamente, ya no existe dicho rechazo por parte de las empresas del sistema financiero, sin embargo, sí es cierto que están invirtiendo en tecnología y fidelización del cliente, en tanto es predecible que es cuestión de tiempo antes de que las empresas de tecnología financiera revolucionen el mercado como lo conocemos.

En ese sentido, en mayo de este año, durante la sesión de presentación de la política monetaria del Banco Central de Reserva-BCR, el gerente central de estudios económicos del BCR comentó que esta nueva forma de financiamiento ayudará a elevar la competencia, la inclusión financiera y el Producto Bruto Interno-PBI potencial $y$, con el desarrollo de una mayor competencia, se esperaría una presión a la baja en las tasas de interés del mercado.

La experiencia internacional validaría esta hipótesis. Un caso interesante es el de España, donde los bancos tuvieron que flexibilizar el acceso a sus créditos gracias al desarrollo del financiamiento participativo. Esto se ha evidenciado también en el caso de Brasil, ya que, al tener un sistema financiero altamente burocrático con elevadas tasas de interés a nivel mundial, ha estado sometido a mayor influencia de las fintech en vista que los consumidores requerían mejorar la experiencia financiera y acceder a menores tasas de interés ${ }^{12}$.

En Colombia, la tendencia expectante es que el desarrollo de las fintech impacte en una baja de tasas. En este país el fenómeno para el crédito - que viene por Crowd- puede generar oferta de tasas más atractivas al crédito tradicional. Por su parte, en Chile se dio una mejora en las tasas de interés y en el PBI. En el caso del crowdlending se espera un mayor impacto en el acceso al crédito principalmente, con una reducción medianamente significativa en el costo de financiamiento.

\section{IMPACTO EN LAS TASAS DE INTERÉS}

El dictamen busca que no haya límites para las tasas de interés que cobrarían las fintech de

10. Gemma Ames, «Las Fintech y su incidencia en la inclusión financiera en Perú (2015- 2017)» (tesis para optar por el título profesional de Contador Público, Universidad Nacional Mayor de San Marcos, 2018), 49, https://cybertesis. unmsm.edu.pe/bitstream/handle/cybertesis/10000/Ames_gg.pdf?sequence=1\&isAllowed=y.

11. Eduardo Chipoco Rodríguez, Angel Flores Ascención, Larry Torres Valdivia y Ubaldo Varea López, «Las Fintech en Brasil, Argentina, Chile y Bolivia: Lecciones y buenas prácticas» (trabajo aplicativo final, Pontificia Universidad Católica del Perú, 2018), 3, https://www.centrumthink.pucp.edu.pe/Docs/files/las_fintech_en_brasil,_argentina,_chile_y_bolivia.pdf.

12. «Brasil recupera el liderazgo Fintech en América Latina y supera la barrera de los 370 startups», FINNOVISTA, 2018, https://www.finnovista.com/actualizacion-fintech-radar-brasil-2018/. 
crowdfunding, como ocurre en las entidades del sistema financiero. Sin embargo, para lograr dicho objetivo se requeriría modificar el Código Civil ${ }^{13}$.

Actualmente las fintech no pueden cobrar una tasa de interés mayor a $45.69 \%$ en soles y $8.12 \%$ en dólares, pues de acuerdo con el Código Civil, las empresas no financieras - clasificación en la que entrarían las de crowdfunding - no pueden fijar una tasa mayor a la tasa promedio del sistema financiero para créditos a la microempresa.

Esto cambiaría con el nuevo tope de tasas tanto en soles como en dólares-, establecido por el Banco Central de Reserva en agosto de este año. Éste regirá a partir del primero de octubre del 2019. El nuevo límite sería definido según la tasa promedio del sistema financiero para créditos de consumo, que es más alta que la tasa de créditos a la microempresa.

"El nuevo tope permitirá que estas entidades puedan atender a un segmento al que no ingresaban por la prohibición de cobrar una tasa alineada al riesgo de la operación. La modificación dinamizará la competencia entre entidades no financieras y el sector financiero, compuesto por la banca, cajas financieras y edpymes ${ }^{\prime \prime 4}$.

Al haber mayor competencia para el sector bancario, éste también se vería obligado a reducir sus tasas de interés, las cuales actualmente son fijadas libremente. Éste y otros beneficios - como una indudable inclusión financiera que permita atender las necesidades que hoy no atiende la banca- dinamizarían el sector, con importantes repercusiones en el desarrollo de la economía.

Precisamente es en esta diversificación donde surge un abanico de posibilidades que fomen- tan la inclusión financiera, como ha sucedido en los casos de difusión y capacitación de pequeños empresarios para poder acceder a servicios financieros por medios digitales haciendo que las operaciones sean más rápidas y cómodas, para poder acceder a préstamos mediante crowdfunding, entre otros.

Por otro lado, y como ya se mencionó, establecer capitales mínimos es importante, pero quedará a discreción de la SMV determinar los criterios. "Fijar capitales mínimos tiene que responder al tipo de riesgo que asume la plataforma, que no son similares a los que puede tener un banco. Son negocios totalmente distintos", nos indica Rivero. De este modo, la estructura que respalde una fintech es importante para garantizar las operaciones que realice. Los beneficios, son, finalmente para los usuarios.

\section{REGULACIÓN DE FINTECH EN OTRAS LE- GISLACIONES}

\section{Caso España.}

En el año 2019 se aprobó la Ley 5/2015 de Fomento de la Financiación Empresarial. En ella se establece que todas las plataformas de crowdlending de préstamos deben contar con la licencia de Plataforma de Financiación Participativa otorgada por Comisión Nacional del Mercado de Valores - CNMV - para funcionar. Es decir, que solo se exige este requisito a las plataformas que ofrecen préstamos como producto financiero. Asimismo, establece los requisitos financieros que deben cumplir estas empresas, la exigencia de contar con seguros de responsabilidad civil, los requisitos que deben tener los inversores acreditados, entre otros aspectos.

\section{Caso México.}

En marzo de 2018 se promulgó la ley para re-

13. El artículo 1243 del Código Civil establece que "la tasa máxima de interés convencional compensatorio o moratorio, es fijada por el Banco Central de Reserva del Perú" y "cualquier exceso sobre la tasa máxima da lugar a la devolución o a la imputación al capital, la voluntad del deudor".

14. José Fernández, «Nuevo tope de tasas impulsará mercado», SEMANAeconómica, n. 1684 (2019). 
gular las fintech. Esta ley impone requisitos para operar tales como (a) controles internos y administración de riesgos, (b) seguridad de la información y continuidad de la operación, y (c) límites de operación por cada cliente. Asimismo, se ha reforzado la protección al consumidor con el objetivo de mantener la confidencialidad de la información. En ese sentido, se han asignado riesgos y responsabilidades. También se ha nombrado organismos supervisores, se ha establecido una política de cumplimiento de un marco de prevención de la corrupción y lavado de activos, y se han establecido sanciones específicas ante determinados supuestos ${ }^{15}$.

Un punto importante para mencionar es que se estableció como requisito que las fintech cuenten con la autorización de la Comisión Nacional Bancaria y de Valores-CNBV. A la fecha de elaboración del presente artículo — setiembreya venía finalizando el plazo para que las empresas que buscan operar como tales obtengan la autorización correspondiente.

No obstante, no muchas empresas fintech han decido solicitar la autorización, en vista de los altos costos y requisitos exigidos en la norma. Es tanto así que se calcula que de unas 500 empresas que operan normalmente bajo esta modalidad en México, aproximadamente solo el 50\%, como máximo, llegará a solicitar la autorización ${ }^{16}$.

La necesidad y costo de la obtención de dicha autorización conlleva a incurrir en gastos que muchas veces resultan excesivos para pequeñas empresas que están comenzando a posicionarse en el negocio de los servicios tecnológicos financieros. En consecuencia, la exigencia indicada desincentiva en parte a las empresas más pequeñas, no lo suficientemente preparadas con todos los requisitos necesarios, por lo que el número de fintech que podrán operar válidamente en México será menor que el proyectado inicialmente.

Por otro lado, al ser los requisitos más estrictos y los montos de inversión más elevados, solo aquellas empresas fintech verdaderamente interesadas terminarán invirtiendo de todas maneras. De esta forma, se mitiga el riesgo de que empresas poco serias pretendan ser plataformas de financiamiento participativo reduciéndose de esta manera también los riesgos cibernéticos o de fraude, y protegiéndose al consumidor.

\section{Caso Chile y Colombia.}

Si bien en ambos países aún no existe una regulación formal de fintech, sí existe un claro interés del sector por promover su regulación. En Chile, por ejemplo, ya se ha anunciado que este año el Ministerio de Hacienda enviará al Congreso un proyecto de ley Fintech. Por lo que es evidente que para el gobierno chileno ello es de fundamental interés.

Antecedentes de este afán de regular se observan ya en los Lineamientos Generales para la Regulación del Crowdfunding y Servicios Relacionados elaborados por la Comisión para el Mercado Financiero - CMF- en febrero de este año ${ }^{17}$.

En Colombia, más que en una ley fintech, se viene trabajando en una política nacional de Fintech que consista en una coordinación entre el gobierno, el sistema financiero y el ecosistema, con el objetivo de desarrollar mecanismos de

15. Para mayor información ver la Ley para la Regular a las Instituciones de Tecnología Financiera (llamadas fintech) publicada el 9 de marzo de 2018.

16. Antonio Hernandez, 9 de setiembre de 2019, «Ley fintech arrancará con pocas empresas», El Universal, https:// www.eluniversal.com.mx/cartera/ley-fintech-arrancara-con-pocas-empresas.

17. Para mayor información, ver: http://www.cmfchile.cl/portal/principal/605/articles-25860_recurso_9.pdf. 
financiamiento alternativo, y mejorar la conectividad del sistema financiero ${ }^{18}$.

\section{EL DESAFÍO DE LA SMV}

La Superintendencia del Mercado de Valores tiene un rol fundamental en la regulación del mercado de las fintechs en nuestro país. De aprobarse el proyecto de ley presentado, la ley que se promulgue deberá detallar aspectos fundamentales para el funcionamiento de las fintechs. La predictibilidad será fundamental para atraer la inversión.

Las plataformas están preparadas y saben que hay que adecuarse a una regulación. En ese sentido, ésta no representaría un gran riesgo para ellas, teniendo en cuenta además que todavía no hay una inversión considerable de crowdfunding. La razón principal de la regulación es la protección que requiere el que solicita el préstamo y el inversor. Además, como se ha mencionado anteriormente, México y España ya cuentan con una ley, por lo que, pese a que el mercado peruano aún es pequeño, hay una razón de mercado mayor que impulsa su desarrollo.

Así, la SMV tendría la facultad de supervisar a las sociedades administradoras de plataformas tecnológicas, que pueden ser aplicaciones informáticas o medios de comunicación electrónicos o digitales. La SMV deberá verificar la calidad de los proyectos que buscan atraer in- versión. No obstante, en caso una empresa requiera de una autorización para el desarrollo de préstamos, será la Superintendencia de Banca y Seguros-SBS quien deberá emitir una opinión de manera previa. Consideramos adecuado que en esta materia sea la SBS y no la SMV el regulador a cargo.

La SBS intervendría a requerimiento de la SMV y solo con la finalidad de expresar una opinión. Además, de acuerdo con el dictamen, la SMV puede solicitar a la SBS cooperación técnica, según los convenios que se celebren, en el caso que la Sociedad Administradora desarrolle la modalidad de financiamiento participativo a través de préstamos ${ }^{19}$.

Dejar a la SMV un campo excesivo de discrecionalidad podría ser perjudicial para el sector. Los aspectos que más preocupan son la regulación de tasas de interés y el capital mínimo que deben tener las entidades financieras ${ }^{20}$.

Respecto a la regulación de tasas de interés, es importante establecer un régimen distinto con la finalidad de lograr una verdadera inclusión financiera. El dictamen aprobado busca que no haya límites en las tasas de interés de las fintechs de crowdfunding.

En cuanto a la determinación del monto de capital mínimo para la operación de las sociedades administradoras, que también estaría a cargo de la SMV, se espera que los requisitos de capital

18. Eliana Rodríguez, 8 de setiembre de 2020, «Colombia quiere ir más allá de una Ley Fintech», Colombia Fintech, https://www.colombiafintech.co/novedades/colombia-mas-alla-de-una-ley-fintech.

19. Otros aspectos de interés relacionados a SBS en el ámbito del proyecto de ley son los siguientes: - Las empresas supervisadas por la SBS pueden crear subsidiarias que tengan por objeto desarrollar plataformas de financiamiento participativo financiero. En este supuesto serían supervisadas por la SMV, pero las opiniones de la SBS serán vinculantes.

- La SMV y la SBS contarían con capacidades para crear sandbox regulatorios, es decir, espacios de innovación conjuntos entre el sistema financiero y el sistema de valores.

- La SBS puede requerir a las plataformas de financiamiento participativo financiero el registro de la información en la Central de Riesgos que administra.

20. Otro aspecto a tener en cuenta es el establecer límites máximos de recursos a recaudar por proyecto — para financiar actividades-, o el número máximo de veces que el receptor puede realizar ofrecimiento en las plataformas por ejercicio económico. 
mínimo no sean muy altos, sobre todo cuando se trata de impulsar un mercado que aún es incipiente, pues, caso contrario, éste se ralentizaría. Ambos aspectos se desarrollarán más adelante.

En España, por ejemplo, se regula a las plataformas de financiación participativa estableciéndose que éstas deben contar en todo momento con un capital social mínimo de 60,000 euros. Y, cuando la suma de financiación obtenida en los últimos 12 meses por los proyectos publicados en la plataforma supere los 2 millones de euros, deberán disponer de recursos propios que como mínimo sumen 120,000 mil euros.

De los detalles que determine la SMV dependerá si finalmente se genera una mayor dinamización del mercado. Según la Asociación Fintech del Perú, más modelos locales surgirían o adaptarían su modelo con la entrada en vigencia de la nueva ley, siempre que las condiciones sean favorables. Así, el mercado potencial es grande. Se esperaría la entrada de empresas extranjeras que están a la espera de un marco normativo que pueda dotar de mayor seguridad las transacciones y proteger al inversor. Pero también hay fintechs locales que están a la expectativa de una regulación para poder realizar operaciones de crowdfunding.

Lo ideal sería que la SMV tenga una regulación diferenciada según volúmenes de operaciones. No se les debería regular como si fuesen entidades financieras porque éstas no realizan una intermediación financiera, sino operativa.

En ese sentido, las fintech pequeñas deberían tener un régimen menos oneroso, pues si finalmente se exige un capital muy grande, un emprendedor podría decidir colocar su dinero en otro proyecto. Este tipo de barreras irracionales son las que deberían evitarse, así como la propia expedición de las licencias de operación de las fintechs, que podría demorar entre 12 y 16 meses, lo que resulta excesivo para que una empresa empiece a operar.
Hasta el momento el regulador se ha mostrado a favor de promover este tipo de actividades; $y$ esto tiene total sentido, pues si ya se ha emitido un dictamen flexible y general, sería contradictorio emitir un reglamento que la bloquee. " $L a$ idea es que la industria surja con ciertos parámetros mínimos", dijo Carlos Rivero, superintendente adjunto de investigación y desarrollo de la SMV en una entrevista que se realizó para SEMANAeconómica. Esto es positivo y esperemos que realmente sea así.

\section{CONCLUSIONES}

El mercado tecnológico financiero está en crecimiento y es innegable que hay grandes expectativas de desarrollo de las fintech en general y las de crowdfunding en específico y como primer paso. El Perú no es ajeno a ello, por lo que esperamos se apruebe su pronta regulación, como ya se ha venido dando en otras legislaciones.

La futura regulación no debe dejar de lado el hecho de que nos encontramos ante un mercado nuevo que aún está en formación, por lo que los controles y requisitos que se le impongan no necesariamente deben ser los mismos que los de las empresas del sistema financiero. Por el contrario, es obligación del legislador analizar cuidadosamente el mercado financiero y regular las fintech de crowdfunding de manera que no se obstruya su desarrollo.

La iniciativa peruana hasta el momento es positiva. Esperamos poder contar, a corto plazo, con una adecuada regulación que permita el crecimiento del mercado financiero de las fintech en el Perú. El impacto de ello en la inclusión financiera y la probable reducción en las tasas de interés dinamizaría, sin duda, el mercado, lo que repercutiría a largo plazo en una economía más sólida y menos dependiente de factores externos - como la guerra comercial, o incluso, el inestable clima político peruano-. 